\title{
Controllability of abstract fractional differential evolution equations with nonlocal conditions
}

\author{
Haiyong Qin ${ }^{\mathrm{a}, \mathrm{b}}$, Chenghui Zhang ${ }^{\mathrm{a}}$, Tongxing $\mathrm{Li}^{\mathrm{a}, \mathrm{c}, \mathrm{d}, *}$, Ying Chen ${ }^{\mathrm{b}}$ \\ a School of Control Science and Engineering, Shandong University, Jinan, Shandong 250061, P. R. China. \\ ${ }^{b}$ School of Mathematics, Qilu Normal University, Jinan, Shandong 250013, P. R. China. \\ ${ }^{c}$ LinDa Institute of Shandong Provincial Key Laboratory of Network Based Intelligent Computing, Linyi University, Linyi, Shandong \\ 276005, P. R. China. \\ ${ }^{d}$ School of Informatics, Linyi University, Linyi, Shandong 276005, P. R. China.
}

\begin{abstract}
In this paper, the controllability of a class of fractional differential evolution equations with nonlocal conditions is investigated. Sufficient conditions which guarantee the controllability of fractional differential evolution equations are obtained. The method used is the contraction mapping principle and Krasnoselskii theorem. A fractional distributed parameter control system is provided to illustrate the applications of our results. (C)2017 All rights reserved.
\end{abstract}

Keywords: Fractional differential equation, controllability, nonlocal condition, fixed point theorem. 2010 MSC: 26A33, 34A12, 93B05.

\section{Introduction}

Fractional calculus is an area having a long history whose infancy dates back to three hundred years. Later on, with the development of computer technology and fractional calculus theory, fractional differential equations have numerous applications in natural sciences and engineering. For instance, fractional differential equations with nonlocal conditions are often used for modeling various phenomena arising in control, electrochemistry, viscoelasticity, and electromagnetics.

During the past few years, a great deal of interest in existence of solutions to various classes of fractional differential and difference equations has been shown. We refer the reader to the papers [111, 14-17] and the references cited therein. In particular, many authors investigated the existence of mild solutions of fractional differential equations in Banach space by semigroup techniques and fixed point theorems; see, e.g., the papers [4, 5, 7, 9-11, 14, 16, 17]. Thereinto, Shu and Wang [14] studied the existence and uniqueness of mild solutions for a differential equation with nonlocal conditions in a

\footnotetext{
*Corresponding author

Email addresses: qhymath@hotmail.com (Haiyong Qin), litongx2007@163.com (Tongxing Li)
} 
Banach space $\mathbb{X}$

$$
\left\{\begin{array}{l}
{ }^{c} D_{t}^{\alpha} u(t)=A u(t)+f(t, u(t))+\int_{0}^{t} q(t-s) g(s, u(s)) d s, \quad t \in[0, T] \\
u(0)+m(u)=u_{0} \in \mathbb{X}, \quad u^{\prime}(0)+n(u)=u_{1} \in \mathbb{X}
\end{array}\right.
$$

where ${ }^{C} D_{t}^{\alpha}$ is Caputo's fractional derivative of order $1<\alpha<2, A: D(A) \subset \mathbb{X} \rightarrow \mathbb{X}$ is a sectorial operator of type $(M, \theta, \alpha, \mu), f, g:[0, T] \times \mathbb{X} \rightarrow \mathbb{X}$ are continuous functions, and nonlocal maps $m, n: \mathbb{X} \rightarrow \mathbb{X}$ are continuous. The method relies on the fixed point theorems and solution operator theorems. By introducing solution operators, the authors gave a reasonable definition of mild solutions, and they also provided estimates on solution operators which will be needed to study the existence results. Because of the complexity, the analysis of distributed parameter systems is difficult. It is well known that controllability and observability are important for the analysis and design of distributed parameter systems. Under certain assumptions, partial differential equations can be written to ordinary differential equations in a functional space. More precisely, the controllability problem for distributed parameter systems can be transformed into that of lumped parameter systems; see Sakthivel et al. [12, 13].

Inspired by the previous papers and many known results reported in [12-14], we study controllability of a class of fractional differential evolution equations with nonlocal conditions

$$
\left\{\begin{array}{l}
{ }^{c} D_{t}^{\alpha} x(t)=A x(t)+f\left(t, x(t), \int_{0}^{t} h(t, s, x(s)) d s\right)+B u(t), \quad t \in I=[0, b], \\
x(0)+g_{1}(x)=x_{0} \in \mathbb{X}, \quad x^{\prime}(0)+g_{2}(x)=x_{0}^{\prime} \in \mathbb{X}
\end{array}\right.
$$

where ${ }^{C} D_{t}^{\alpha}$ is Caputo's fractional derivative of order $1<\alpha<2, A: D(A) \subset \mathbb{X} \rightarrow \mathbb{X}$ is a sectorial operator of type $(M, \theta, \alpha, \mu), f: I \times \mathbb{X} \times \mathbb{X} \rightarrow \mathbb{X}$ and $h: \Delta \times \mathbb{X} \rightarrow \mathbb{X}$ are continuous functions, $\Delta=\{(\mathrm{t}, \mathrm{s}) \in \mathrm{I} \times \mathrm{I}, 0 \leqslant$ $\mathrm{t} \leqslant \mathrm{s} \leqslant \mathrm{b}\}$, nonlocal maps $\mathrm{g}_{1}, \mathrm{~g}_{2}: \mathbb{X} \rightarrow \mathbb{X}$ are continuous, $\mathrm{B}: \mathrm{U} \rightarrow \mathbb{X}$ is a bounded linear operator, control function $u(\cdot) \in \mathrm{L}^{2}(\mathrm{I}, \mathrm{U}), \mathrm{U}$ is a Banach space, and $\mathbb{X}$ is a Banach space endowed with the norm $\|\cdot\|$.

Equation (1.2) has a more general form than equation (1.1). Nonlocal conditions $x(0)+g_{1}(x)=x_{0}$ and $x^{\prime}(0)+g_{2}(x)=x_{0}^{\prime}$ are more realistic than the local ones in treating physical problems. The problem considered in this paper has a strong physical background; see, for instance, fractional integrodifferential equations appeared in the study of dynamical systems when the controlled systems are described by fractional equations.

The remainder of this paper is organized as follows. In Section 2, we present some necessary definitions and lemma that will be used to prove our main results, and we also introduce a suitable definition of mild solution of fractional evolution equation (1.2). The main results are given in Section 3. Finally, in Section 4, an example is provided to demonstrate the effectiveness of our results.

\section{Preliminaries}

We need the following basic definitions and properties from the fractional calculus.

Definition 2.1. The fractional integral of order $\gamma$ with the lower limit zero for a function $f$ is defined as

$$
I^{\gamma} f(t)=\frac{1}{\Gamma(\gamma)} \int_{0}^{t}(t-s)^{\gamma-1} f(s) d s, \quad t>0, \quad \gamma>0,
$$

provided that the right side is point-wise defined on $[0,+\infty)$, where $\Gamma(\cdot)$ is the gamma function.

Definition 2.2. The Caputo derivative of the order $\gamma$ for a function $f \in \mathrm{C}^{\mathrm{n}}[0, \infty)$ is defined by

$$
{ }^{c} D^{\gamma} f(t)=\frac{1}{\Gamma(n-\gamma)} \int_{0}^{t}(t-s)^{n-\gamma-1} f^{(n)}(s) d s=I^{n-r} f^{(n)}(t), \quad t>0, \quad n-1<\gamma<n .
$$


Remark 2.3. If $\mathrm{f}$ is an abstract function with values in $\mathbb{X}$, then integrals which appear in Definitions 2.1 and 2.2 are taken in Bochner's sense.

Definition 2.4 ([14]). Let $A: D(A) \subseteq \mathbb{X} \rightarrow \mathbb{X}$ be a closed linear operator. $A$ is said to be a sectorial operator of type $(M, \theta, \alpha, \mu)$ if there exist $0<\theta<\pi / 2, M>0$, and $\mu \in \mathbb{R}$ such that the $\alpha$-resolvent of $A$ exists outside the sector

$$
\mu+S_{\theta}=\left\{\mu+\lambda^{\alpha}: \lambda \in \mathbb{C},\left|\operatorname{Arg}\left(-\lambda^{\alpha}\right)\right|<\theta\right\}
$$

and

$$
\left\|\left(\lambda^{\alpha} I-A\right)^{-1}\right\| \leqslant \frac{M}{\left|\lambda^{\alpha}-\mu\right|}, \quad \lambda^{\alpha} \notin \mu+S_{\theta} .
$$

Compared with the fractional differential equation (1.1), we introduce a reasonable concept of mild solutions for fractional evolution system (1.2) which describes a more general form. In what follows, we use the notation $(H x)(t)=\int_{0}^{t} h(t, s, x(s)) d s$, unless mentioned otherwise.

Lemma 2.5. Let $A$ be a sectorial operator of type $(M, \theta, \alpha, \mu)$. If $f$ satisfies a uniform Hölder condition with exponent $\beta \in(0,1]$, then the mild solutions of (1.2) are fixed points of the operator equation

$$
(\mathrm{Q} x)(\mathrm{t})=\mathcal{S}_{\alpha}(\mathrm{t})\left(\mathrm{x}_{0}-\mathrm{g}_{1}(\mathrm{x})\right)+\mathcal{K}_{\alpha}(\mathrm{t})\left(\mathrm{x}_{0}^{\prime}-\mathrm{g}_{2}(\mathrm{x})\right)+\int_{0}^{\mathrm{t}} \mathcal{T}_{\alpha}(\mathrm{t}-\mathrm{s})[\mathrm{f}(\mathrm{s}, \mathrm{x}(\mathrm{s}),(\mathrm{Hx})(\mathrm{s}))+\mathrm{Bu}(\mathrm{s})] \mathrm{d} \mathrm{s},
$$

where

$$
\mathcal{S}_{\alpha}(t)=\frac{1}{2 \pi i} \int_{c} e^{\lambda t} \lambda^{\alpha-1} R\left(\lambda^{\alpha}, A\right) d \lambda, \quad \mathcal{K}_{\alpha}(t)=\frac{1}{2 \pi i} \int_{c} e^{\lambda t} \lambda^{\alpha-2} R\left(\lambda^{\alpha}, A\right) d \lambda,
$$

and

$$
\mathcal{T}_{\alpha}(t)=\frac{1}{2 \pi i} \int_{c} e^{\lambda t} R\left(\lambda^{\alpha}, A\right) d \lambda
$$

with $\mathrm{c}$ being a suitable path such that $\lambda^{\alpha} \notin \mu+\mathrm{S}_{\theta}$ for $\lambda \in \mathrm{c}$.

The proof of Lemma 2.5 is similar to that of [14, Theorems 3.1 and 3.2]. For more details about sectorial operators of type $(M, \theta, \alpha, \mu)$, one can refer to [14]. On the basis of the estimates on $\mathcal{S}_{\alpha}(t), \mathcal{K}_{\alpha}(t)$, and $\mathcal{T}_{\alpha}(t)$ (see [14, Theorems 3.3 and 3.4] for details), it is not difficult to see that $\mathcal{S}_{\alpha}(t), \mathcal{K}_{\alpha}(t)$, and $\mathcal{T}_{\alpha}(t)$ have the following results which will be used later.

Proposition 2.6. Operators $\mathcal{S}_{\alpha}(\mathrm{t}), \mathcal{K}_{\alpha}(\mathrm{t})$, and $\mathcal{T}_{\alpha}(\mathrm{t})$ have the following properties:

(1) there exists a constant $M_{1}>0$ such that

$$
\sup _{t \in I}\left\|\mathcal{S}_{\alpha}(t)\right\| \leqslant M_{1}, \quad \sup _{t \in I}\left\|\mathcal{K}_{\alpha}(t)\right\| \leqslant M_{1}, \quad \sup _{t \in I}\left\|\mathcal{T}_{\alpha}(t)\right\| \leqslant M_{1} ;
$$

(2) for all $\epsilon>0$ and $t_{1}, t_{2} \in(0, b]$, there exists a constant $\delta>0$ such that for $\left|t_{1}-t_{2}\right|<\delta$,

$$
\left\|\mathcal{S}_{\alpha}\left(t_{1}\right)-\mathcal{S}_{\alpha}\left(t_{2}\right)\right\| \leqslant \epsilon, \quad\left\|\mathcal{K}_{\alpha}\left(t_{1}\right)-\mathcal{K}_{\alpha}\left(t_{2}\right)\right\| \leqslant \epsilon, \quad\left\|\mathcal{T}_{\alpha}\left(t_{1}\right)-\mathcal{T}_{\alpha}\left(t_{2}\right)\right\| \leqslant \epsilon .
$$

Definition 2.7. System (1.2) is said to be controllable on interval $[0, b]$ if, for every $x_{0}, y_{0} \in \overline{D(A)}$, there exists a control $u \in L^{2}(I, U)$ such that a mild solution $x$ of $(1.2)$ satisfies $x(b)+g_{1}(x)=y_{0}$.

Theorem 2.8 (Krasnoselskii theorem). Assume that $\mathrm{D}$ is a closed convex and nonempty subset of a Banach space $\mathbb{X}$. Let $\mathrm{Q}_{1}$ and $\mathrm{Q}_{2}$ be two operators such that

(1) $\mathrm{Q}_{1} \mathrm{x}_{1}+\mathrm{Q}_{2} \mathrm{x}_{2} \in \mathrm{D}$ whenever $\mathrm{x}_{1}, \mathrm{x}_{2} \in \mathrm{D}$;

(2) $\mathrm{Q}_{1}$ is a contraction mapping;

(3) $\mathrm{Q}_{2}$ is compact and continuous.

Then there exists a $z \in \mathrm{D}$ such that $z=\mathrm{Q}_{1} z+\mathrm{Q}_{2} z$. 


\section{Main results}

In order to demonstrate the main results, we list the following reasonable hypotheses.

$\left(\mathrm{H}_{1}\right)$ For any $\mathrm{u}_{1}, \mathrm{u}_{2}, v_{1}, v_{2} \in \mathbb{X}$, there exist three functions $\mu_{1}, \mu_{2}, v_{1} \in \mathrm{L}\left(\mathrm{I}, \mathbb{R}^{+}\right)$such that

$$
\left\|f\left(t, u_{1}, v_{1}\right)-f\left(t, u_{2}, v_{2}\right)\right\| \leqslant \mu_{1}(t)\left\|u_{1}-u_{2}\right\|+\mu_{2}(t)\left\|v_{1}-v_{2}\right\|
$$

and $\left\|h\left(t, s, u_{1}\right)-h\left(t, s, u_{2}\right)\right\| \leqslant v_{1}(t)\left\|u_{1}-u_{2}\right\|$.

$\left(H_{2}\right) g_{1}, g_{2}: \mathbb{X} \rightarrow \overline{\mathrm{D}(\mathrm{A})}$ are continuous. For any $u_{1}, u_{2} \in \mathbb{X}$, there exist two constants $\mathrm{N}_{1}$ and $\mathrm{N}_{2}$ such that $\left\|g_{1}\left(u_{1}\right)-g_{1}\left(u_{2}\right)\right\| \leqslant N_{1}\left\|u_{1}-u_{2}\right\|$ and $\left\|g_{2}\left(u_{1}\right)-g_{2}\left(u_{2}\right)\right\| \leqslant N_{2}\left\|u_{1}-u_{2}\right\|$.

$\left(H_{3}\right)$ The linear operator $W$ from $L^{2}(I, U)$ into $\mathbb{X}$ defined by $W u=\int_{0}^{b} \mathcal{T}_{\alpha}(b-s) B u(s) d s$ induces an invertible operator $W^{-}$defined on $\mathrm{L}^{2}(\mathrm{I}, \mathrm{U}) / \mathrm{KerW}$, and there exists a constant $\mathrm{K}>0$ such that $\left\|B W^{-}\right\| \leqslant K$.

$\left(\mathrm{H}_{4}\right)$ For any $k>0$, there exists a function $\mu_{k} \in L\left(I, \mathbb{R}^{+}\right)$such that $\sup _{\|x\| \leqslant k}\|f(t, x,(H x))\| \leqslant \mu_{k}(t)$.

Theorem 3.1. Suppose that hypotheses $\left(\mathrm{H}_{1}\right)-\left(\mathrm{H}_{3}\right)$ hold. Then system (1.2) is controllable on I provided that

$$
\frac{\mathrm{L}-\mathrm{KN}}{\mathrm{K}}+\mathrm{M}_{1} \mathrm{Lb}<1,
$$

where

$$
L=K N_{1}+K M_{1} N_{1}+K M_{1} N_{2}+K M_{1}\left(\int_{0}^{b} \mu_{1}(s) d s+\int_{0}^{b} \mu_{2}(s) d s \times \int_{0}^{b} v_{1}(s) d s\right) .
$$

Proof. Using $\left(\mathrm{H}_{3}\right)$, for an arbitrary function $x(\cdot)$, we define the control $u$ and operator $\mathrm{Q}: \mathrm{C}(\mathrm{I}, \mathbb{X}) \rightarrow \mathrm{C}(\mathrm{I}, \mathbb{X})$ by

$$
u(t)=W^{-}\left[y_{0}-g_{1}(x)-\mathcal{S}_{\alpha}(b)\left(x_{0}-g_{1}(x)\right)-\mathcal{K}_{\alpha}(b)\left(x_{0}^{\prime}-g_{2}(x)\right)-\int_{0}^{b} \mathcal{T}_{\alpha}(b-s) f(s, x(s),(H x)(s)) d s\right](t)
$$

and

$$
(\mathrm{Qx})(\mathrm{t})=\mathcal{S}_{\alpha}(\mathrm{t})\left(\mathrm{x}_{0}-\mathrm{g}_{1}(\mathrm{x})\right)+\mathcal{K}_{\alpha}(\mathrm{t})\left(\mathrm{x}_{0}^{\prime}-\mathrm{g}_{2}(\mathrm{x})\right)+\int_{0}^{\mathrm{t}} \mathcal{T}_{\alpha}(\mathrm{t}-\mathrm{s})[\mathrm{f}(\mathrm{s}, \mathrm{x}(\mathrm{s}),(\mathrm{H} \mathrm{x})(\mathrm{s}))+\mathrm{Bu}(\mathrm{s})] \mathrm{d} \mathrm{s},
$$

respectively. For any $x_{1}, x_{2} \in C(I, \mathbb{X})$, by $\left(H_{1}\right)-\left(H_{3}\right)$ and (3.2), we get

$$
\begin{aligned}
\left\|B u_{1}(t)-B u_{2}(t)\right\| \leqslant & \| B W^{-}\left[y_{0}-g_{1}\left(x_{1}\right)-\mathcal{S}_{\alpha}(b)\left(x_{0}-g_{1}\left(x_{1}\right)\right)\right. \\
& \left.-\mathcal{K}_{\alpha}(b)\left(x_{0}^{\prime}-g_{2}\left(x_{1}\right)\right)-\int_{0}^{b} \mathcal{T}_{\alpha}(b-s) f\left(s, x_{1}(s),\left(H x_{1}\right)(s)\right) d s\right](t) \\
& -B W^{-}\left[y_{0}-g_{1}\left(x_{2}\right)-\mathcal{S}_{\alpha}(b)\left(x_{0}-g_{1}\left(x_{2}\right)\right)\right. \\
& \left.-\mathcal{K}_{\alpha}(b)\left(x_{0}^{\prime}-g_{2}\left(x_{2}\right)\right)-\int_{0}^{b} \mathcal{T}_{\alpha}(b-s) f\left(s, x_{2}(s),\left(H x_{2}\right)(s)\right) d s\right](t) \| \\
\leqslant & K\left\|g_{1}\left(x_{1}\right)-g_{1}\left(x_{2}\right)\right\|+K M_{1}\left\|g_{1}\left(x_{1}\right)-g_{1}\left(x_{2}\right)\right\|+K M_{1}\left\|g_{2}\left(x_{1}\right)-g_{2}\left(x_{2}\right)\right\| \\
& +K M_{1} \int_{0}^{b} \mu_{1}(s)\left\|x_{1}-x_{2}\right\| d s+K M_{1} \int_{0}^{b} \mu_{2}(s)\left\|\left(H x_{1}\right)(s)-\left(H x_{2}\right)(s)\right\| d s \\
\leqslant & \left(K N_{1}+K M_{1} N_{1}+K M_{1} N_{2}\right)\left\|x_{1}-x_{2}\right\| \\
& +K M_{1}\left(\int_{0}^{b} \mu_{1}(s) d s+\int_{0}^{b} \mu_{2}(s) d s \times \int_{0}^{b} v_{1}(s) d s\right)\left\|x_{1}-x_{2}\right\| \\
\leqslant & L\left\|x_{1}-x_{2}\right\|
\end{aligned}
$$


and

$$
\begin{aligned}
\|\left(Q x_{1}\right)(t)- & \left(Q x_{2}\right)(t) \| \\
\leqslant & \| \mathcal{S}_{\alpha}(t)\left(x_{0}-g_{1}\left(x_{1}\right)\right)+\mathcal{K}_{\alpha}(t)\left(x_{0}^{\prime}-g_{2}\left(x_{1}\right)\right)+\int_{0}^{t} \mathcal{T}_{\alpha}(t-s)\left[f\left(s, x_{1}(s),\left(H x_{1}\right)(s)\right)+B u_{1}(s)\right] d s \\
& -\mathcal{S}_{\alpha}(t)\left(x_{0}-g_{1}\left(x_{2}\right)\right)-\mathcal{K}_{\alpha}(t)\left(x_{0}^{\prime}-g_{2}\left(x_{2}\right)\right)-\int_{0}^{t} \mathcal{T}_{\alpha}(t-s)\left[f\left(s, x_{2}(s),\left(H x_{2}\right)(s)\right)+B u_{2}(s)\right] d s \| \\
\leqslant & \left(\frac{L-K N_{1}}{K}+M_{1} L b\right)\left\|x_{1}-x_{2}\right\| .
\end{aligned}
$$

By virtue of (3.1), the operator $\mathrm{Q}$ is a contraction mapping, and so $\mathrm{Q}$ has a fixed point. It follows from $\left(\mathrm{H}_{3}\right)$ that the fixed point is a mild solution of control problem (1.2) and $x(b)+g_{1}(x)=y_{0}$. Therefore, system (1.2) is controllable on I.

Theorem 3.2. Let hypotheses $\left(\mathrm{H}_{2}\right)-\left(\mathrm{H}_{4}\right)$ be satisfied and suppose that

$$
M_{1}\left(N_{1}+N_{2}\right)<1
$$

and

$$
\begin{aligned}
& M_{1}\left(\left\|x_{0}\right\|+\left\|x_{0}^{\prime}\right\|+N_{1} r+\left\|g_{1}(0)\right\|+N_{2} r+\left\|g_{2}(0)\right\|\right) \\
& \quad+b M_{1} K\left(\left\|y_{0}\right\|+N_{1} r+\left\|g_{1}(0)\right\|+M_{1}\left\|x_{0}\right\|+M_{1} N_{1} r+M_{1}\left\|g_{1}(0)\right\|+M_{1}\left\|x_{0}^{\prime}\right\|\right. \\
& \left.\quad+M_{1} N_{2} r+M_{1}\left\|g_{2}(0)\right\|+M_{1} \int_{0}^{b} \mu_{r}(s) d s\right)<r
\end{aligned}
$$

for some constant $r>0$. Then system (1.2) is controllable on I.

Proof. Set $\mathrm{B}_{\mathrm{r}}=\{x \in \mathbb{X}:\|x\| \leqslant r\}$. For $x \in \mathrm{B}_{\mathrm{r}}$, define the operator $\mathrm{Q}=\mathrm{Q}_{1}+\mathrm{Q}_{2}$, where

$$
\left(Q_{1} x\right)(t)=\mathcal{S}_{\alpha}(t)\left(x_{0}-g_{1}(x)\right)+\mathcal{K}_{\alpha}(t)\left(x_{0}^{\prime}-g_{2}(x)\right)
$$

and

$$
\left(Q_{2} x\right)(t)=\int_{0}^{t} \mathcal{T}_{\alpha}(t-s)[f(s, x(s),(H x)(s))+B u(s)] d s
$$

Then

$$
\left\|\left(Q_{1} x\right)(t)\right\| \leqslant M_{1}\left\|x_{0}-g_{1}(x)\right\|+M_{1}\left\|x_{0}^{\prime}-g_{2}(x)\right\| \leqslant M_{1}\left(\left\|x_{0}\right\|+\left\|x_{0}^{\prime}\right\|+N_{1} r+\left\|g_{1}(0)\right\|+N_{2} r+\left\|g_{2}(0)\right\|\right)
$$

and

$$
\begin{aligned}
\left\|\left(Q_{2} y\right)(t)\right\| \leqslant & M_{1} \int_{0}^{t}\left\|f\left(s, y(s), \int_{0}^{s} h(s, \tau, y(\tau)) d \tau\right)\right\| d s+M_{1} \int_{0}^{t}\|B u(s)\| d s \\
\leqslant & M_{1} \int_{0}^{t} \mu_{r}(s) d s+M_{1} \int_{0}^{t}\|B u(s)\| d s \\
\leqslant & M_{1} \int_{0}^{b} \mu_{r}(s) d s+b M_{1} k\left(\left\|y_{0}\right\|+N_{1} r+\left\|g_{1}(0)\right\|+M_{1}\left\|x_{0}\right\|+M_{1} N_{1} r+M_{1}\left\|g_{1}(0)\right\|\right. \\
& \left.+M_{1}\left\|x_{0}^{\prime}\right\|+M_{1} N_{2} r+M_{1}\left\|g_{2}(0)\right\|+M_{1} \int_{0}^{b} \mu_{r}(s) d s\right) .
\end{aligned}
$$

Using (3.4), we deduce that $\left\|\left(Q_{1} x\right)(t)+\left(Q_{2} y\right)(t)\right\| \leqslant r$. That is, for any $x, y \in B_{r}, Q_{1} x+Q_{2} y \in B_{r}$. Next, for any $x, y \in B_{r}$, we have

$$
\begin{aligned}
\left\|\left(Q_{1} x\right)(t)-\left(Q_{1} y\right)(t)\right\| & \leqslant\left\|\mathcal{S}_{\alpha}(t)\left(x_{0}-g_{1}(x)\right)+\mathcal{K}_{\alpha}(t)\left(x_{0}^{\prime}-g_{2}(x)\right)-\mathcal{S}_{\alpha}(t)\left(x_{0}-g_{1}(y)\right)-\mathcal{K}_{\alpha}(t)\left(x_{0}^{\prime}-g_{2}(y)\right)\right\| \\
& \leqslant M_{1}\left\|g_{1}(y)-g_{1}(x)\right\|+M_{1}\left\|g_{2}(y)-g_{2}(x)\right\|
\end{aligned}
$$




$$
\begin{aligned}
& \leqslant M_{1}\left\|g_{1}(y)-g_{1}(x)\right\|+M_{1}\left\|g_{2}(y)-g_{2}(x)\right\| \\
& \leqslant M_{1} N_{1}\|y-x\|+M_{1} N_{2}\|y-x\| \\
& =M_{1}\left(N_{1}+N_{2}\right)\|y-x\| .
\end{aligned}
$$

It follows from (3.3) that $\mathrm{Q}_{1}$ is a contraction mapping.

Let $\left\{x_{n}\right\}_{n=1}^{\infty}$ be a sequence in $B_{r}, x \in B_{r}$, and $x_{n} \rightarrow x(n \rightarrow \infty)$. Noting that $f$ is continuous on $\mathrm{I} \times \mathbb{X} \times \mathbb{X}$, we get

$$
f\left(s, x_{n}(s),\left(H x_{n}\right)(s)\right) \rightarrow f(s, x(s),(H x)(s)), \quad n \rightarrow \infty .
$$

For all $\mathrm{t} \in[0, \mathrm{~b}]$, we obtain

$$
\begin{aligned}
\left\|\left(\mathrm{Q}_{2} x_{n}\right)(t)-\left(\mathrm{Q}_{2} x\right)(t)\right\| \leqslant & \| \int_{0}^{t} \mathcal{T}_{\alpha}(t-s)\left[f\left(s, x_{n}(s),\left(H x_{n}\right)(s)\right) d s+B u_{n}(s)\right] d s \\
& -\int_{0}^{t} \mathcal{T}_{\alpha}(t-s)[f(s, x(s),(H x)(s)) d s+B u(s)] d s \| .
\end{aligned}
$$

Define $u$ as in Theorem 3.1. Then

$$
\begin{aligned}
\left\|\left(B u_{n}\right)(t)-(B u)(t)\right\| \leqslant & \| B W^{-}\left[y_{0}-g_{1}\left(x_{n}\right)-\mathcal{S}_{\alpha}(b)\left(x_{0}-g_{1}\left(x_{n}\right)\right)-\mathcal{K}_{\alpha}(b)\left(x_{0}^{\prime}-g_{2}\left(x_{n}\right)\right)\right. \\
& \left.-\int_{0}^{b} \mathcal{T}_{\alpha}(b) f\left(s, x_{n}(s),\left(H x_{n}\right)(s)\right) d s\right](t) \\
& -B W^{-}\left[y_{0}-g_{1}(x)-\mathcal{S}_{\alpha}(b)\left(x_{0}-g_{1}(x)\right)-\mathcal{K}_{\alpha}(b)\left(x_{0}^{\prime}-g_{2}(x)\right)\right. \\
& \left.-\int_{0}^{b} \mathcal{T}_{\alpha}(b) f(s, x(s),(H x)(s)) d s\right](t) \| \\
\leqslant & K\left\|g_{1}(x)-g_{1}\left(x_{n}\right)\right\|+K\left\|\mathcal{S}_{\alpha}(b) g_{1}(x)-\mathcal{S}_{\alpha}(b) g_{1}\left(x_{n}\right)\right\| \\
& +K\left\|\mathcal{K}_{\alpha}(b) g_{2}(x)-\mathcal{K}_{\alpha}(b) g_{2}\left(x_{n}\right)\right\| \\
& +K \int_{0}^{b} \mathcal{T}_{\alpha}(b-s)\left\|f(s, x(s),(H x)(s))-f\left(s, x_{n}(s),\left(H x_{n}\right)(s)\right)\right\| d s \\
\leqslant & K N_{1}\left\|x_{n}-x\right\|+K M_{1} N_{1}\left\|x_{n}-x\right\|+K M_{1} N_{2}\left\|x_{n}-x\right\| \\
& +K_{1} \int_{0}^{b}\left\|f(s, x(s),(H x)(s))-f\left(s, x_{n}(s),\left(H x_{n}\right)(s)\right)\right\| d s .
\end{aligned}
$$

By (3.6) and the Lebesgue dominated convergence theorem, it is easy to see that

$$
\left\|\left(Q_{2} x_{n}\right)(t)-\left(Q_{2} x\right)(t)\right\| \rightarrow 0, \quad n \rightarrow \infty .
$$

Thus we conclude that $\mathrm{Q}_{2}$ is continuous. In order to present the compactness of $\mathrm{Q}_{2}$, we prove that $\left\{\left(Q_{2} x\right)(t): x \in B_{r}\right\}$ is relatively compact for all $t \in I$ and uniformly bounded, respectively. It follows from (3.5) that $\left\|\left(Q_{2} x\right)(t)\right\| \leqslant C$, where $C$ is a constant. For $0<t_{1}<t_{2} \leqslant b$, we obtain

$$
\begin{aligned}
\left\|\left(Q_{2} x\right)\left(t_{1}\right)-\left(Q_{2} x\right)\left(t_{2}\right)\right\|= & \| \int_{0}^{t_{2}} \mathcal{T}_{\alpha}\left(t_{1}-s\right)[f(s, x(s),(H x)(s))+B u(s)] d s \\
& +\int_{t_{2}}^{t_{1}} \mathcal{T}_{\alpha}\left(t_{1}-s\right)[f(s, x(s),(H x)(s))+B u(s)] d s \\
& -\int_{0}^{t_{2}} \mathcal{T}_{\alpha}\left(t_{2}-s\right)[f(s, x(s),(H x)(s))+B u(s)] d s \| \\
\leqslant & \int_{0}^{t_{2}}\left\|\mathcal{T}_{\alpha}\left(t_{1}-s\right)-\mathcal{T}_{\alpha}\left(t_{2}-s\right)\right\|\|f(s, x(s),(H x)(s))+B u(s)\| d s
\end{aligned}
$$




$$
\begin{aligned}
& \quad+\int_{\mathrm{t}_{2}}^{\mathrm{t}_{1}}\left\|\mathcal{T}_{\alpha}\left(\mathrm{t}_{1}-\mathrm{s}\right)\right\|\|\mathrm{f}(\mathrm{s}, x(\mathrm{~s}),(\mathrm{H} x)(\mathrm{s}))+\mathrm{Bu}(\mathrm{s})\| \mathrm{ds} \\
& \leqslant \\
& \leqslant
\end{aligned}
$$

where

$$
\mathrm{I}_{1}=\int_{0}^{\mathrm{t}_{2}}\left\|\mathcal{T}_{\alpha}\left(\mathrm{t}_{1}-\mathrm{s}\right)-\mathcal{T}_{\alpha}\left(\mathrm{t}_{2}-\mathrm{s}\right)\right\|\|\mathrm{f}(\mathrm{s}, x(\mathrm{~s}),(\mathrm{H} x)(\mathrm{s}))+\mathrm{Bu}(\mathrm{s})\| \mathrm{ds}
$$

and

$$
\mathrm{I}_{2}=\int_{\mathrm{t}_{2}}^{\mathrm{t}_{1}}\left\|\mathcal{T}_{\alpha}\left(\mathrm{t}_{1}-\mathrm{s}\right)\right\|\|f(s, x(\mathrm{~s}),(\mathrm{H} x)(\mathrm{s}))+\mathrm{Bu}(\mathrm{s})\| \mathrm{ds} .
$$

Noting that the continuity of the function $t \mapsto\left\|\mathcal{T}_{\alpha}(t)\right\|$ for $t \in(0, b]$, we have $\lim _{t_{2} \rightarrow t_{1}} I_{i}=0$. Hence, by the Arzelà-Ascoli theorem, $\mathrm{Q}_{2}$ is compact. Then Definition 2.7 and Theorem 2.8 allow us to conclude that (1.2) is controllable on I.

\section{Example}

In order to demonstrate applications of our main results obtained in Section 3, we consider the following fractional order distributed parameter control system

$$
\left\{\begin{array}{l}
\frac{\partial^{\alpha} u(t, x)}{\partial t^{\alpha}}=\frac{\partial^{2} u(t, x)}{\partial x^{2}}+f\left(t, u(t, x), \int_{0}^{t} h(t, s, u(s, x)) d s\right)+B \mu(t, x), \\
u(t, 0)=u(t, \pi)=0, \quad u^{\prime}(t, 0)=u^{\prime}(t, \pi)=0, \\
u(t, 0)+\sum_{i=1}^{k} a_{i} u\left(t_{i}, x\right)=u_{0}(x), \quad u^{\prime}(t, 0)+c_{1} u\left(t_{1}, x\right)=u_{1}(x),
\end{array}\right.
$$

where $t \in I=[0, b], t_{i} \in(0, b), i=1,2, \ldots, k, x \in[0, \pi], 1<\alpha<2$, and $B: U \rightarrow \mathbb{X}$ is a bounded linear operator. Let $\mathbb{X}=\mathrm{L}^{2}([0, \pi])$ and define the operator $A: D(A) \subseteq \mathbb{X} \rightarrow \mathbb{X}$ by

$$
A(u)=\frac{\partial^{2} u}{\partial x^{2}}, \quad D(A)=\left\{u \in \mathbb{X}, \frac{\partial u}{\partial x}, \frac{\partial^{2} u}{\partial x^{2}} \in \mathbb{X}\right\} .
$$

Obviously, $\mathbb{A}$ is densely defined in $\mathbb{X}$ and is the infinitesimal generator of a resolvent family $\left\{\mathcal{T}_{\alpha}(t)\right\}_{t} \geqslant 0$ on $\mathbb{X}$. For $u, v \in C(I, \mathbb{X})$, define

$$
\begin{aligned}
f(t, u, H u) & =\frac{|u(t, x)|}{\left(6+e^{t}\right)(1+|u(t, x)|)}+\frac{1}{10+e^{t}} \int_{0}^{t} \frac{e^{2 s}}{2 \sqrt{2}+|u(s, x)|} d s, \\
f(t, v, H v) & =\frac{|v(t, x)|}{\left(6+e^{t}\right)(1+|v(t, x)|)}+\frac{1}{10+e^{t}} \int_{0}^{t} \frac{e^{2 s}}{2 \sqrt{2}+|v(s, x)|} d s, \\
g_{1}(u)(x) & =\sum_{i=1}^{k} a_{i} u\left(t_{i}, x\right), \quad \text { and } g_{2}(u)(x)=c_{1} u\left(t_{1}, x\right) .
\end{aligned}
$$

Then we conclude that fractional integrodifferential equation (1.2) serves as an abstract formulation of fractional distributed parameter control system (4.1),

$$
\begin{aligned}
\|f(t, u, H u)-f(t, v, H v)\| \leqslant & \frac{1}{6+e^{t}}\left\|\frac{|u(t, x)|}{1+|u(t, x)|}-\frac{|v(t, x)|}{1+|v(t, x)|}\right\| \\
& +\frac{1}{10+e^{t}}\left\|\int_{0}^{t} \frac{e^{2 s}}{2 \sqrt{2}+|u(s, x)|} d s-\int_{0}^{t} \frac{e^{2 s}}{2 \sqrt{2}+|v(s, x)|} d s\right\| \\
\leqslant & \frac{1}{6}\|u-v\|+\frac{1}{10}\|H u-H v\|,
\end{aligned}
$$


and

$$
\|h(t, s, u)-h(t, s, v)\| \leqslant e^{2 t}\left\|\frac{1}{2 \sqrt{2}+|u(t, x)|}-\frac{1}{2 \sqrt{2}+|v(t, x)|}\right\| \leqslant \frac{e^{2 t}}{8}\|u-v\| \leqslant \frac{e^{2 b}}{8}\|u-v\|
$$

Hence, we can choose $\mu_{1}(t)=1 / 6, \mu_{2}(t)=1 / 10, v_{1}(t)=e^{2 b} / 8, N_{1}=\sum_{i=1}^{k} a_{i}$, and $N_{2}=c_{1}$. Assume now that $\left(\mathrm{H}_{3}\right)$ holds. With the choices of $b, K$, and $M_{1}$, inequality (3.1) can be satisfied. Therefore, by Theorem 3.1, system (1.2) is controllable on I, and thus fractional distributed parameter control system (4.1) is controllable on I.

\section{Acknowledgment}

The authors express their sincere gratitude to the editors and two anonymous referees for useful comments that helped to improve the presentation of the results and accentuate important details. This research is supported by Major International (Regional) Joint Research Project of the National Natural Science Foundation of China (Grant No. 61320106011), National Natural Science Foundation of China (Grant Nos. 61527809 and 61503171), China Postdoctoral Science Foundation (Grant No. 2015M582091), Shandong Provincial Natural Science Foundation (Grant Nos. ZR2016AB04 and ZR2016JL021), Foundation for Young Teachers of Qilu Normal University (Grant No. 2016L0605), Doctoral Scientific Research Foundation of Linyi University (Grant No. LYDX2015BS001), and Scientific Research Foundation for University Students of Qilu Normal University (Grant No. XS2015L03).

\section{References}

[1] B. Ahmad, J. J. Nieto, Riemann-Liouville fractional integro-differential equations with fractional nonlocal integral boundary conditions, Bound. Value Probl., 2011 (2011), 9 pages. 1

[2] B. Ahmad, S. Sivasundaram, On four-point nonlocal boundary value problems of nonlinear integro-differential equations of fractional order, Appl. Math. Comput., 217 (2010), 480-487.

[3] Z.-B. Bai, On positive solutions of a nonlocal fractional boundary value problem, Nonlinear Anal., 72 (2010), 916-924.

[4] K. Balachandran, J. Y. Park, Nonlocal Cauchy problem for abstract fractional semilinear evolution equations, Nonlinear Anal., 71 (2009), 4471-4475. 1

[5] K. Balachandran, J. J. Trujillo, The nonlocal Cauchy problem for nonlinear fractional integrodifferential equations in Banach spaces, Nonlinear Anal., 72 (2010), 4587-4593. 1

[6] Y.-K. Chang, J. J. Nieto, Existence of solutions for impulsive neutral integro-differential inclusions with nonlocal initial conditions via fractional operators, Numer. Funct. Anal. Optim., 30 (2009), 227-244.

[7] C. S. Goodrich, Existence and uniqueness of solutions to a fractional difference equation with nonlocal conditions, Comput. Math. Appl., 61 (2011), 191-202. 1

[8] L.-Y. Hu, Y. Ren, R. Sakthivel, Existence and uniqueness of mild solutions for semilinear integro-differential equations of fractional order with nonlocal initial conditions and delays, Semigroup Forum, 79 (2009), 507-514.

[9] F. Li, J. Liang, H.-K. Xu, Existence of mild solutions for fractional integrodifferential equations of Sobolev type with nonlocal conditions, J. Math. Anal. Appl., 391 (2012), 510-525. 1

[10] G. M. Mophou, G. M. N'Guerékata, Existence of the mild solution for some fractional differential equations with nonlocal conditions, Semigroup Forum, 79 (2009), 315-322.

[11] G. M. N'Guérékata, A Cauchy problem for some fractional abstract differential equation with non local conditions, Nonlinear Anal., 70 (2009), 1873-1876. 1

[12] R. Sakthivel, N. I. Mahmudov, J. J. Nieto, Controllability for a class of fractional-order neutral evolution control systems, Appl. Math. Comput., 218 (2012), 10334-10340. 1

[13] R. Sakthivel, Y. Ren, N. I. Mahmudov, On the approximate controllability of semilinear fractional differential systems, Comput. Math. Appl., 62 (2011), 1451-1459. 1

[14] X.-B. Shu, Q.-Q. Wang, The existence and uniqueness of mild solutions for fractional differential equations with nonlocal conditions of order $1<\alpha<2$, Comput. Math. Appl., 64 (2012), 2100-2110. 1, 1, 2.4, 2

[15] X.-M. Zhang, X.-Y. Huang, Z.-H. Liu, The existence and uniqueness of mild solutions for impulsive fractional equations with nonlocal conditions and infinite delay, Nonlinear Anal. Hybrid Syst., 4 (2010), 775-781.

[16] Y. Zhou, F. Jiao, Existence of mild solutions for fractional neutral evolution equations, Comput. Math. Appl., 59 (2010), 1063-1077. 1

[17] Y. Zhou, F. Jiao, Nonlocal Cauchy problem for fractional evolution equations, Nonlinear Anal. Real World Appl., 11 (2010), 4465-4475. 1 